\title{
Intracoronary Near-Infrared Spectroscopy (NIRS) Imaging for Detection of Lipid Content of Coronary Plaques: Current Experience and Future Perspectives
}

\author{
Milosz Jaguszewski • Roland Klingenberg • \\ Ulf Landmesser \\ Published online: 17 August 2013 \\ (C) The Author(s) 2013. This article is published with open access at Springerlink.com
}

\begin{abstract}
Acute coronary syndromes are frequently caused by "vulnerable" coronary plaques with a lipid-rich core. In 1993 near-infrared spectroscopy (NIRS) was first used to detect the lipid (cholesterol) content of atherosclerotic plaques in an experimental animal study. NIRS was then carefully validated using human atherosclerotic plaques (ex vivo), and has subsequently been developed for intracoronary imaging in humans, for which now an FDA-approved catheter-based NIRS system is available. NIRS provides a "chemogram" of the coronary artery wall and is used to detect lipid-rich plaques. Using this technology, recent studies have shown that lipid-rich plaques are very frequent in the culprit lesion of patients with an acute coronary syndrome, and are also common in non-culprit coronary lesions in these patients as compared to patients with stable coronary disease. First studies are evaluating the impact of statin therapy on coronary NIRS-detected lipid cores. Intracoronary NIRS imaging represents a highly interesting method for coronary plaque characterization in humans and may become a valuable tool for the development of novel therapies aiming to impact on the biology of human coronary artery plaques, likely in combination with other intracoronary imaging techniques, such as optical coherence tomography.
\end{abstract}

Keywords Intracoronary near-infrared spectroscopy (NIRS) Imaging $\cdot$ Lipid content of coronary plaques $\cdot$ Acute coronary syndromes $\cdot$ Chemogram $\cdot$ Coronary lesions

\section{Introduction}

Atherosclerotic plaques with a large lipid core are a frequent cause of acute coronary syndromes [1]. In 1993 near-infrared

M. Jaguszewski $\cdot$ R. Klingenberg $\cdot$ U. Landmesser $(\bowtie)$

Department of Cardiology, University Heart Center, University

Hospital Zurich, Raemistrasse 100, 8091 Zurich, Switzerland

e-mail: Ulf.Landmesser@usz.ch spectroscopy (NIRS) was first used for atherosclerotic plaque imaging in an experimental animal model [2]. In further ex vivo validation studies, NIRS was found to accurately detect the lipid (cholesterol) content of human atherosclerotic plaques [3]. In 2001, a device prototype for intracoronary imaging was developed [4]. Recently, NIRS received US Food and Drug Administration approval [5]. NIRS provides a "chemogram" of the wall of the coronary artery and aims to detect lipid-rich plaques [6].

\section{NIRS Catheter}

A commercially available catheter-based NIRS imaging system has been developed. This NIRS imaging system consists of a 3.2 French catheter (InfraReDx, Burlington, Massachusetts, USA), a pullback, rotation device, and a dedicated console comprising a laser light source and a computer for algorithmic data processing [7]. The catheter comprises the rotating core with optical fibers that deliver and collect near-infrared light [7]. The light is emitted into a sample by the NIR spectrometer, which subsequently measures the proportion of light that is returned over the range of optical wavelength [6]. The NIRS system uses wave lengths of 800 to $2500 \mathrm{~nm}$, converting a diffuse reflectance signal from an object to produce a spectrum. Subsequently, a computer analyzes the spectra and produces an algorithm to demonstrate a chemogram, that corresponds strongly to the lipid content [5] (Figs. 1 and 2).

\section{Experience Using NIRS for Coronary Plaque Imaging in Humans in Vivo}

Non-invasive imaging modalities including computed tomography or magnetic resonance have a limited resolution for precise plaque characterization [5]. Therefore, catheter-based intravascular imaging methods, such as optical coherence 
Fig. 1 An example of nearinfrared spectroscopy (NIRS). A 'chemogram' is shown detecting a colour map of the artery wall and indicates the location and intensity of coronary lipid content. The horizontal-axis of the chemogram represents the pullback position and the verticalaxis represents the circumferential position in degrees $\left(0-360^{\circ}\right)$. Adapted and modified from: Choi $B$ et al. Eur Heart J 2013;34:2047-2054
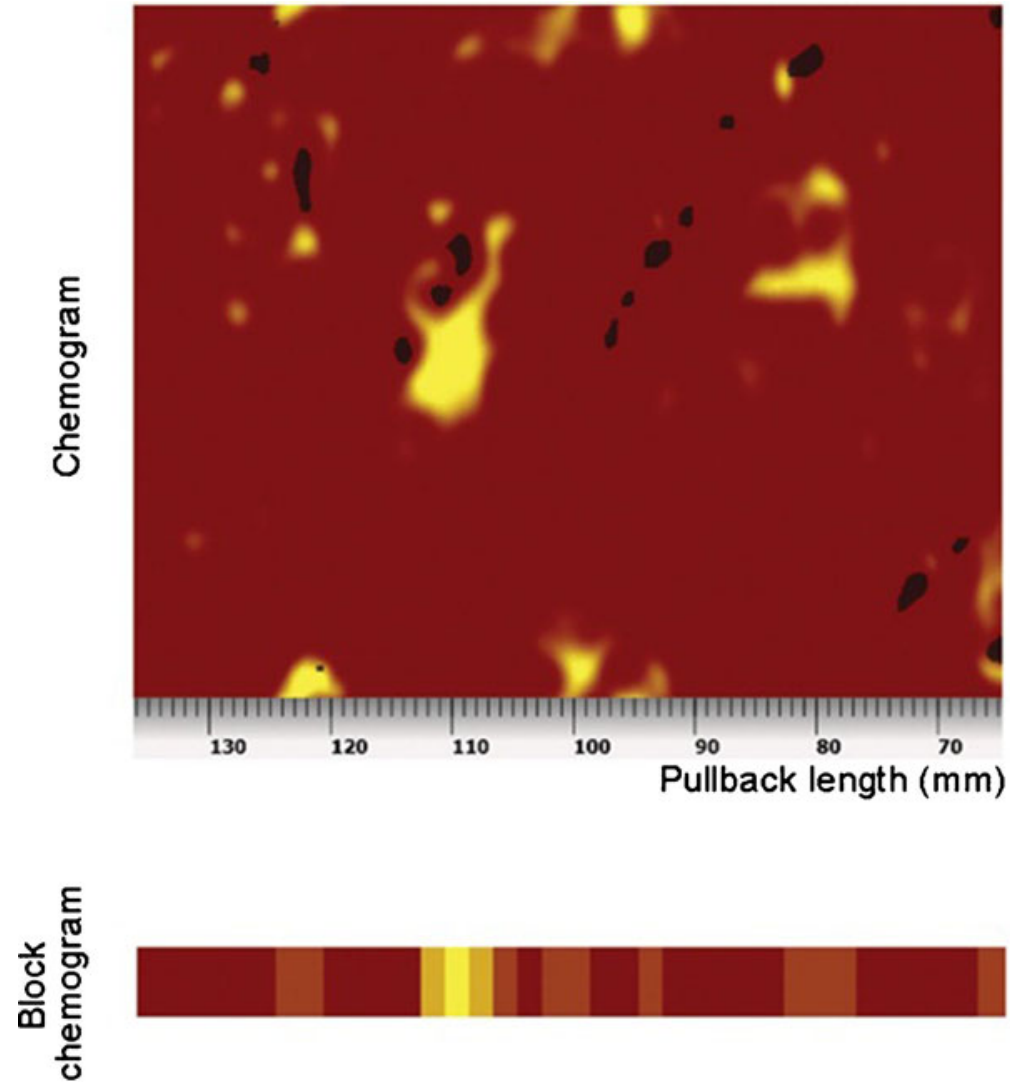

High probability

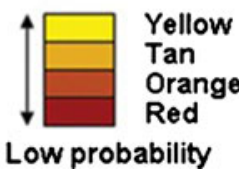

tomography (OCT) and NIRS are highly valuable for detailed characterization of coronary atherosclerotic plaques, and have become available within the last years.

As described above, the NIRS imaging system allowing for plaque assessment was carefully validated against atherosclerotic plaque autopsy specimens, and can nowadays be used in humans in vivo [7, 8]. A summary of recent clinical investigations is outlined in Table 1. NIRS provides excellent spectra through blood and despite heart movement by using short scanning acquisition cycles [7]. The PROSPECT study suggested, that a plaque burden of $>70 \%$, thin-cap fibroatheroma, and minimal luminal area $\leq 4.0 \mathrm{~mm}^{2}$ are predictors of overall long-term adverse cardiovascular events [9]. NIRS has been established as a very accurate modality to characterize plaques with different lipid content [8]. Madder et al. have shown, that target lesions responsible for acute coronary syndrome are frequently composed of lipid core plaque with a high lipid core burden index (LCBI) [10]. The presence of lipid core plaque may address the high-risk setting for subsequent coronary events, which needs to be further investigated in future studies $[11,12]$. Interestingly, both culprit and non-culprit lesions contained lipid core plaque more frequently in ACS patients as compared to patients with stable angina [10].
Moreover, the study by Pu et al. have recently supported the notion, that combining NIRS with IVUS can lead to a better plaque characterization [13]. In addition, the three-dimensional reconstruction of coronary anatomy by ANGIOCARE Software permits the identification of the lipid core plaque location and therefore the association among vessel geometry, endothelial shear stress and plaque composition [14].

\section{Implications for Guiding Therapy}

The impact of short-term intensive statin treatment on a lipid plaque content has been investigated in a first study using NIRS demonstrating changes in lipid composition [15]. Several small studies have examined the potential impact of plaque evaluation by NIRS for interventional coronary therapy [16-18]. It is well known, that 3-15\% of percutaneous coronary interventions (PCIs) are complicated by periprocedural myocardial infarction (MI) due to the distal embolization by intraluminal thrombus and/or lipid-core plaque content [19-21]. First studies suggest that edge dissections or plaque disruptions may be more common when stents end within a lipid core plaque [22]. Dixon et al. have documented, that in $16 \%$ of lesions lipid core plaque may extend 
Fig. 2 Near-infrared spectroscopy (NIRS) imaging indicates the lipid-core containing plaques are substantially more frequent in human coronary arteries with endothelial dysfunction. Adapted and modified from: Choi B et al. Eur Heart J 2013;34:2047-2054
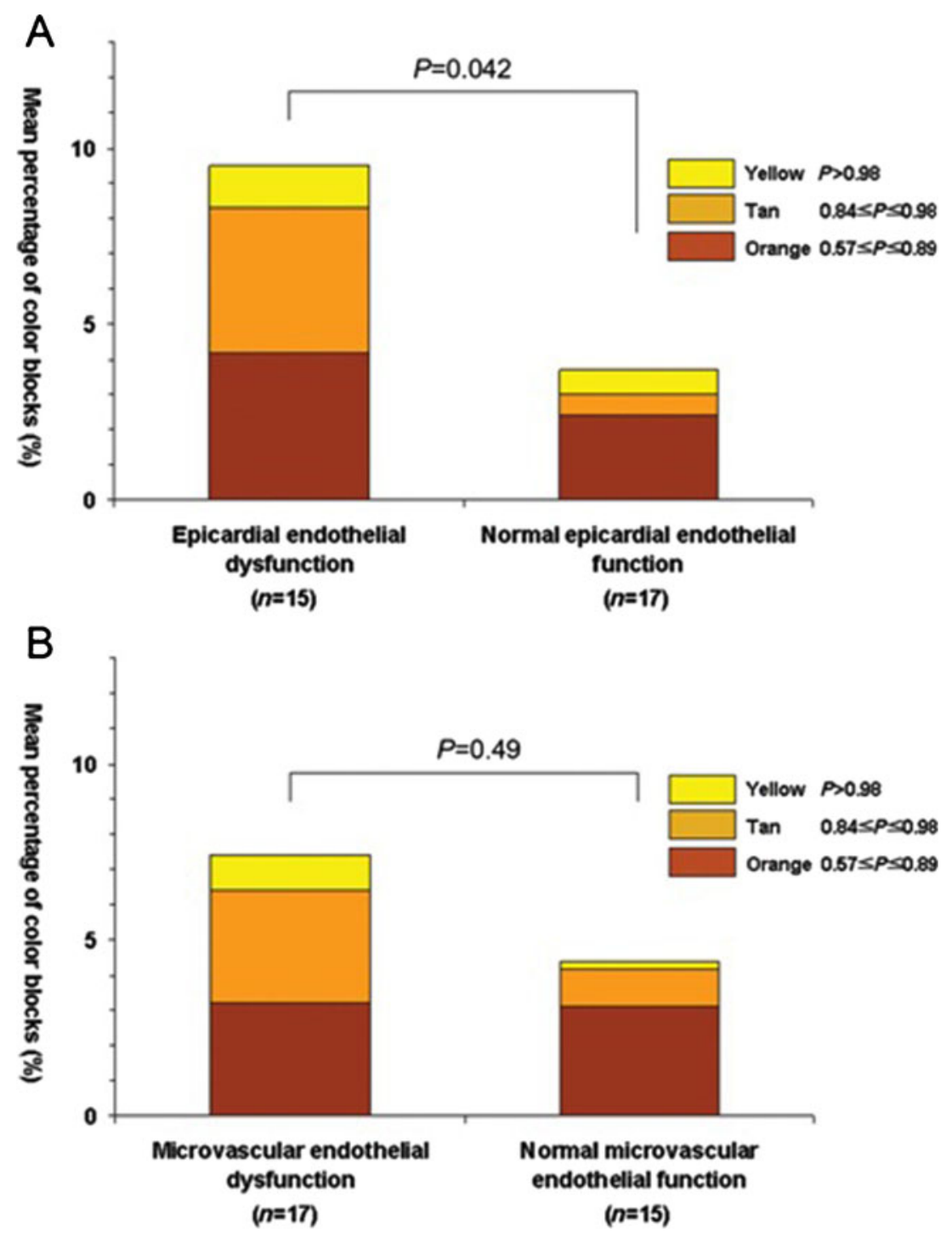

beyond the margins of the lesion as assessed by angiography [23]. This further points to limitations of QCA alone in the evaluation of lesion- and subsequently stent length [23]. Notably, the NIRS-identified lipid core plaque has been described to be strongly associated with a high risk of thrombus formation or periprocedural myocardial infarction [17, 18, 24].

\section{Future Perspectives}

NIRS is likely to become a sensitive modality for coronary plaque characterization. NIRS could be an interesting tool to investigate novel lipid-modulating and other cardiovascular therapies aiming to prevent adverse coronary events [17, 23]. However, a potential limitation of NIRS may be its inability to assess the depth of a lipid core and the measurement of lipid volume has not been validated so far [5]. NIRS may therefore be used in combination with other imaging modalities, such as IVUS or OCT. In this respect, a combined imaging catheter has recently been developed (TVC Imaging System, MC 7 system, InfraReDx, Burlington, Massachusetts, USA), combining both, an IVUS probe and NIRS light source. This catheter is being used in the IBIS-3 (Integrated Biomarker and Imaging Study 3) to investigate the effects of rosuvastatin therapy on the lipidrich coronary atherosclerotic plaques [25]. 
Table 1 Intracoronary human NIRS studies

\begin{tabular}{lll}
\hline Study & Year & $\mathrm{n}^{*}$ p \\
\hline $\begin{array}{l}\text { Caplan J.D. et .al [6] } \\
\text { JACC }\end{array}$ & 2006 & 6 \\
$\begin{array}{l}\text { Waxman, S. et .al [7] } \\
\text { JACC Cardiovasc Imaging }\end{array}$ & 2009 & 106 \\
& & \\
Raghunathan, D. et .al [17] & 2011 & 30 \\
Am J Cardiol & & \\
$\begin{array}{l}\text { Goldstein, J.A. et .al [18] } \\
\text { Circ Cardiovasc Interv }\end{array}$ & 2011 & 62 \\
$\begin{array}{l}\text { Brugaletta, S. et .al [26] } \\
\text { JACC Cardiovasc Imaging }\end{array}$ & 2011 & 31
\end{tabular}

Madder, R.D. et .al [10] 201260

Circ Cardiovasc Interv

$\mathrm{Pu}$, J. et .al [13]

201266

Eur Heart $J$

Dixon, S.R. et .al [23]

Am J Cardiol

Brugaletta, S. et .al [27]

2012202

JACC Cardiovasc Imaging

Brilakis, E.S. et .al [16]

Catheter Cardiovasc

Interv

Brugaletta, S. et .al [28]

201268

JACC Cardiovasc Imaging

Kini, A.S. et .al [15]

$J A C C$

Papayannis, A.C. et .al [24] 20139

Catheter Cardiovasc

Interv

Townsend, J.C. et .al [29] $2013 \quad 100$

Am J Cardiol

Maini, A. et .al [30]

201377

J Inv Cardiol

Madder, R.D. et .al [31]

201320

JACC Cardiovasc Interv

201378

Zynda, T.K. et .al [32]

Catheter Cardiovasc

Interv

Choi, B.J. et .al [33]

Eur Heart $J$

$2013 \quad 32$
Initial clinical experience in stable CAD

To determine whether catheter-based NIRS signals from coronaries of patients are similar to those from autopsy specimens and to assess initial safety of NIRS device

To examine whether an association exists between the presence and extent of LCP detected by NIRS performed before $\mathrm{PCI}$ with postprocedural MI

To analyze the relationship between the presence of a large LCP detected by NIRS and periprocedural MI

To compare the findings of NIRS, IVUS virtual histology and grayscale IVUS obtained in matched coronary vessel segments of patients undergoing coronary angiography

To determine the frequency of LCP at target and remote sites in ACS vs. stable angina

To evaluate NIRS combined with IVUS to provide novel information of human coronary plaque characterization

To compare the target lesion length using NIRS combined with angiography vs. angiography alone

To explore a relationship between lipid plaque composition by NIRS and angiographic severity of coronary artery disease

To investigate whether use of an embolic protection device might prevent complications of LCP interventions

To assess LCP distribution in nonculprit coronary arteries using NIRS

To determine the impact of short-term intensive statine treatment on intracoronary plaque lipid content

To examine the association between presenting LCP (by NIRS) and poststenting thrombus formation (by OCT)

To investigate, whether coronary bifurcations have higher levels of intracoronary LCP than nonbifurcation regions

To evaluate LCP modification with coronary revascularization and its correlation with periprocedural MI

To describe NIRS findings of culprit lesions in STEMI

To determine if there was a relationship between angiographic lesion complexity and the extent of LCP identified by catheter-based NIRS

To investigate whether coronary endothelial dysfunction is associated with the LCP in patients with early CAD

Conclusion

High quality NIRS spectra can be obtained in patients in vivo

Spectral data were safely obtained by NIRS similarly to those from autopsy specimens; results demonstrated the feasibility of invasive detection of coronary LCP

PCI of LCP-positive lesions is associated with increased risk for MI after PCI

NIRS provides rapid, automated detection of extensive LCPs that are associated with a high risk of periprocedural MI

Larger plaque area by grayscale IVUS was more often associated with either elevated percentage VH necrotic core or LCP by NIRS

Target lesion responsible for ACS were frequently composed of LCP; LCPs often were found in remote, non-target areas; LCPs were more common in patients with ACS vs. stable angina patients

Combining NIRS and IVUS contributes to the plaque characterization

Patients undergoing stent implantation could have LCP extending beyond the intended treatment margins as defined using QCA alone

Patients with highest Syntax score have a higher LCBI

Use of embolic protection devices frequently resulted in embolized material retrieval after stenting of native coronary artery lesions with large LCP

LCP were mainly located in proximal portions of the LAD and LCX, and more uniformly distributed in the RCA;

Short-term intensive treatment with statine may reduce lipid content in obstructive coronary lesions

Stenting large LCPs may be associated with intrastent thrombus formation

Coronary bifurcations do not appear to have higher levels of intracoronary LCP than their comparative non-bifurcation regions

Plaque modification may be performed successfully using interventional methods and can be evaluated with NIRS; axial plaque shifting is an acute prognostic marker for postprocedure MI

Plaques causing STEMI have a high LCBI

Angiographic SYNTAX score weakly correlated with lipid core burden index

Patients with early CAD and endothelial dysfunction had a higher lipid content in the vascular wall than patients with normal endothelial function;

$A C S$ acute coronary syndrome; $C A D$ coronary artery disease; IVUS intravascular ultrasound; $L A D$ left artery descending; $L C B I$ lipid core burden index; $L C P$ lipid core-containing plaque; $L C X$ left circumflex artery; $M I$ myocardial infarction; $N I R S$ near-infrared spectroscopy; $P C I$ percutaneous coronary intervention; $Q C A$ Quantitative Coronary Angiography; RCA right coronary artery; STEMI ST-segment elevation myocardial infarction; *number of patients 


\section{Compliance with ethics Guidelines}

Conflicts of Interest Dr. Milosz Jaguszewski and Dr. Roland Klingenberg reported no conflicts of interest relevant to this article.

Dr. Ulf Landmesser serves as a Section Editor for Current Cardiovascular Imaging Reports.

Human and Animal Rights and Informed Consent This article does not contain any studies with human or animal subjects performed by any of the authors.

Open Access This article is distributed under the terms of the Creative Commons Attribution License which permits any use, distribution, and reproduction in any medium, provided the original author(s) and the source are credited.

\section{References}

1. Libby P. Mechanisms of acute coronary syndromes and their implications for therapy. N Engl J Med. 2013;368:2004-13.

2. Cassis LA, Lodder RA. Near-ir imaging of atheromas in living arterial tissue. Anal Chem. 1993;65:1247-56.

3. Wang J, Geng YJ, Guo B, Klima T, Lal BN, et al. Near-infrared spectroscopic characterization of human advanced atherosclerotic plaques. J Am Coll Cardiol. 2002;39:1305-13.

4. Moreno PR, Muller JE. Identification of high-risk atherosclerotic plaques: a survey of spectroscopic methods. Curr Opin Cardiol. 2002;17:638- 47.

5. Jang IK. Near infrared spectroscopy: another toy or indispensible diagnostic tool? Circ Cardiovasc Interv. 2012;5:10-1.

6. Caplan JD, Waxman S, Nesto RW, Muller JE. Near-infrared spectroscopy for the detection of vulnerable coronary artery plaques. J Am Coll Cardiol. 2006;47:C92-6.

7. Waxman S, Dixon SR, L'Allier P, Moses JW, Petersen JL, et al. In vivo validation of a catheter-based near-infrared spectroscopy system for detection of lipid core coronary plaques: Initial results of the spectacl study. JACC Cardiovasc Imaging. 2009;2:858-68.

8. Gardner CM, Tan H, Hull EL, Lisauskas JB, Sum ST, et al. Detection of lipid core coronary plaques in autopsy specimens with a novel catheter-based near-infrared spectroscopy system. JACC Cardiovasc Imaging. 2008;1:638-48.

9. Stone GW, Maehara A, Lansky AJ, de Bruyne B, Cristea E, et al. A prospective natural-history study of coronary atherosclerosis. N Engl J Med. 2011;364:226-35.

10. Madder RD, Smith JL, Dixon SR, Goldstein JA. Composition of target lesions by near-infrared spectroscopy in patients with acute coronary syndrome versus stable angina. Circ Cardiovasc Interv. 2012;5:55-61.

11. Naghavi M, Libby P, Falk E, Casscells SW, Litovsky S, et al. From vulnerable plaque to vulnerable patient: a call for new definitions and risk assessment strategies: part I. Circulation. 2003;108:1664-72.

12. Naghavi M, Libby P, Falk E, Casscells SW, Litovsky S, et al. From vulnerable plaque to vulnerable patient: a call for new definitions and risk assessment strategies: part II. Circulation. 2003;108:1772-8.

13. Pu J, Mintz GS, Brilakis ES, Banerjee S, Abdel-Karim AR, et al. In vivo characterization of coronary plaques: novel findings from comparing greyscale and virtual histology intravascular ultrasound and near-infrared spectroscopy. Eur Heart J. 2012;33:372-83.

14. Wentzel JJ, van der Giessen AG, Garg S, Schultz C, Mastik F, et al. In vivo $3 \mathrm{~d}$ distribution of lipid-core plaque in human coronary artery as assessed by fusion of near infrared spectroscopy-intravascular ultrasound and multislice computed tomography scan. Circ Cardiovasc Imaging. 2010;3:e6-7.

15. Kini AS, Baber U, Kovacic JC, Limaye A, Ali ZA, et al. Changes in plaque lipid content after short-term intensive versus standard statin therapy: the yellow trial (reduction in yellow plaque by aggressive lipid-lowering therapy). J Am Coll Cardiol. 2013;62:21-9.

16. Brilakis ES, Abdel-Karim AR, Papayannis AC, Michael TT, Rangan $\mathrm{BV}$, et al. Embolic protection device utilization during stenting of native coronary artery lesions with large lipid core plaques as detected by nearinfrared spectroscopy. Catheter Cardiovasc Interv. 2012;80:1157-62.

17. Raghunathan D, Abdel-Karim AR, Papayannis AC, daSilva M, Jeroudi $\mathrm{OM}$, et al. Relation between the presence and extent of coronary lipid core plaques detected by near-infrared spectroscopy with postpercutaneous coronary intervention myocardial infarction. Am J Cardiol. 2011;107:1613-8.

18. Goldstein JA, Maini B, Dixon SR, Brilakis ES, Grines CL, et al. Detection of lipid-core plaques by intracoronary near-infrared spectroscopy identifies high risk of periprocedural myocardial infarction. Circ Cardiovasc Interv. 2011;4:429-37.

19. Niccoli G, Burzotta F, Galiuto L, Crea F. Myocardial no-reflow in humans. J Am Coll Cardiol. 2009;54:281-92.

20. Prasad A, Herrmann J. Myocardial infarction due to percutaneous coronary intervention. N Engl J Med. 2011;364:453-64.

21. Heusch G, Kleinbongard P, Bose D, Levkau B, Haude M, et al. Coronary microembolization: from bedside to bench and back to bedside. Circulation. 2009;120:1822-36.

22. Saeed B, Banerjee S, Brilakis ES. Slow flow after stenting of a coronary lesion with a large lipid core plaque detected by nearinfrared spectroscopy. EuroIntervention. 2010;6:545.

23. Dixon SR, Grines CL, Munir A, Madder RD, Safian RD, et al. Analysis of target lesion length before coronary artery stenting using angiography and near-infrared spectroscopy versus angiography alone. Am J Cardiol. 2012;109:60-6.

24. Papayannis AC, Abdel-Karim AR, Mahmood A, Rangan BV, Makke $\mathrm{LB}$, et al. Association of coronary lipid core plaque with intrastent thrombus formation: a near-infrared spectroscopy and optical coherence tomography study. Catheter Cardiovasc Interv. 2013;81:488-93.

25. Simsek C, Garcia-Garcia HM, van Geuns RJ, Magro M, Girasis C, et al. The ability of high dose rosuvastatin to improve plaque composition in non-intervened coronary arteries: rationale and design of the integrated biomarker and imaging study-3 (ibis-3). EuroIntervention. 2012;8:235-41.

26. Brugaletta S, Garcia-Garcia HM, Serruys PW, de Boer S, Ligthart J, et al. Nirs and ivus for characterization of atherosclerosis in patients undergoing coronary angiography. JACC Cardiovasc Imaging. 2011;4:647-55.

27. Brugaletta S, Magro M, Simsek C, Heo JH, de Boer S, et al. Plaque compositional syntax score: combining angiography and lipid burden in coronary artery disease. JACC Cardiovasc Imaging. 2012;5:S119-21.

28. Brugaletta S, Garcia-Garcia HM, Serruys PW, Gomez-Lara J, de Boer S, et al. Distance of lipid core-rich plaques from the ostium by nirs in nonculprit coronary arteries. JACC Cardiovasc Imaging. 2012;5:297-9.

29. Townsend JC, Steinberg DH, Nielsen CD, Todoran TM, Patel CP, et al. Comparison of lipid deposition at coronary bifurcations versus at nonbifurcation portions of coronary arteries as determined by nearinfrared spectroscopy. Am J Cardiol. 2013;112:369-72.

30. Maini A, Buyantseva L, Maini B. In vivo lipid core plaque modification with percutaneous coronary revascularization: a near-infrared spectroscopy study. J Invasive Cardiol. 2013;25:293-5.

31. Madder RD, Goldstein JA, Madden SP, Puri R, Wolski K, et al. Detection by near-infrared spectroscopy of large lipid core plaques at culprit sites in patients with acute st-segment elevation myocardial infarction. JACC Cardiovasc Interv. 2013. doi:10.1016/j.jcin.2013.04.012.

32. Zynda TK, Thompson CD, Hoang KC, Seto AH, Glovaci D, et al. Disparity between angiographic coronary lesion complexity and lipid core plaques assessed by near-infrared spectroscopy. Catheter Cardiovasc Interv. 2013;81:529-37.

33. Choi BJ, Prasad A, Gulati R, Best PJ, Lennon RJ, et al. Coronary endothelial dysfunction in patients with early coronary artery disease is associated with the increase in intravascular lipid core plaque. Eur Heart J. 2013;34:2047-54. 